\title{
Relevance of pharmacokinetics and pharmacodynamics of inhaled corticosteroids to asthma
}

\author{
H. Derendorf*, R. Nave ${ }^{\#}$, A. Drollmann ${ }^{\#}$, F. Cerasoli ${ }^{\mp}$ and W. Wurst ${ }^{\#}$
}

ABSTRACT: The pharmacokinetic and pharmacodynamic effects of inhaled corticosteroids (ICS) have shaped the efficacy and safety of these agents in the treatment of asthma.

Important pharmacokinetic and pharmacodynamic characteristics that can enhance the efficacy of ICS include small particle size, high glucocorticoid-receptor-binding affinity, long pulmonary residence time and lipid conjugation. These characteristics can increase or prolong the antiinflammatory effects of an ICS. Important pharmacokinetic characteristics that can enhance the safety of ICS include on-site activation in the lung, low oropharyngeal exposure, negligible oral bioavailability, high protein-binding and rapid systemic clearance.

The degree of oropharyngeal exposure is relevant to local side-effects, such as oropharyngeal candidiasis, dysphonia and coughing. Pharmacokinetic properties that influence the degree of systemic exposure are relevant to the pharmacodynamic effect of ICS-induced hypothalamicpituitary-adrenal axis suppression and cortisol suppression, an indicator of potential long-term systemic side-effects, such as reduced growth velocity and bone density, fractures, and skin bruising and thinning.

Therefore, significant differences in the pharmacokinetic and pharmacodynamic characteristics of the currently available inhaled corticosteroids warrant careful consideration when used in clinical practice as they may result in differences in efficacy and local and systemic safety profiles.

KEYWORDS: Ciclesonide, clinical relevance, cortisol, lipid conjugation, on-site activation, side-effects

nhaled corticosteroids (ICS) are the treatment of choice for persistent asthma. Asthma is an inflammatory disease, and ICS are the most effective anti-inflammatory medications available. Clinical studies have shown that ICS significantly reduce airway inflammation and hyperresponsiveness, improve lung function, decrease symptom severity, and effectively prevent or reduce the occurrence of acute asthma exacerbations [1]. National and international guidelines for asthma management currently recommend low-dose ICS as first-line therapy for patients with mild persistent asthma and medium-dose ICS or combination therapy with long-acting $\beta_{2}$-agonists as the preferred therapy for moderate asthma [2]. Combination therapy with high doses of ICS is recommended only for patients with severe persistent asthma that is inadequately controlled by combination therapy with a medium dose of ICS.

Corticosteroids are effective in the treatment of asthma because of their ability to interfere with the multiple pathways involved in the inflammatory process $[3,4]$. Corticosteroids bind within the cell nucleus to regulators that control the transcription of various pro-inflammatory gene products and, therefore, are effective in controlling asthma by reducing the inflammation caused by these multiple mediators. Specifically, the mechanism of action of corticosteroids is believed to begin with diffusion across the cell membrane and subsequent binding to glucocorticoid receptors residing in the cytoplasm of target cells [5]. This binding results in an activated glucocorticoid-receptor-corticosteroid complex that
AFFILIATIONS

\#ALTANA Pharma, Konstanz,

Germany.

*Dept of Pharmaceutics, University of

Florida College of Pharmacy,

Gainesville, $\mathrm{FL}$, and

'ALTANA Pharma, Florham Park, NJ. USA.

\section{CORRESPONDENCE}

H. Derendorf

Dept of Pharmaceutics

University of Florida

100494 College of Pharmacy

Gainesville

FL 32610

USA

Fax: 13523923249

E-mail: hartmut@cop.ufl.edu

Received:

June 272005

Accepted after revision:

June 212006 
subsequently translocates across the nuclear membrane and binds to specific DNA sequences [6]. As a result, gene transcription and protein synthesis are altered. Corticosteroids reduce airway inflammation and hyperresponsiveness by altering the production of mediators associated with inflammatory cells in the airways, such as macrophages, eosinophils, lymphocytes, mast cells and dendritic cells [7]. The reduction in airway hyperresponsiveness has been associated with clinically significant improvement in asthma symptoms [8].

The preferred route of administration of a corticosteroid for the treatment of asthma is inhalation, which delivers the drug directly to the lung, where it acts locally and minimises the systemic side-effects associated with oral or parenteral administration. Unfortunately, ICS can cause both local and systemic side-effects (table 1) [9]. The incidence of local side-effects commonly associated with ICS, such as oropharyngeal candidiasis, dysphonia, reflex cough, bronchospasm and pharyngitis, can be variable $[9,10]$. The occurrence of these side-effects is dependent on both the type and dose of ICS used and the mode of delivery (e.g. metered-dose inhaler (MDI) or dry-powder inhaler (DPI)). For example, dysphonia characterised by hoarseness or voice alterations has been reported in $1-58 \%$ of patients and is dependent upon multiple factors, such as ICS and type of inhaler used, ICS dose, and vocal stress $[11,12]$. Although local side-effects associated with ICS do not lead to significant morbidity, they diminish compliance, leading to uncontrolled asthma and reduced patient quality of life $[13,14]$.

Systemic side-effects associated with ICS can be more serious. Potential systemic side-effects include osteoporosis, reduced growth velocity in children, skin thinning, cataracts and glaucoma, all of which accompany ICS suppression of hypothalamic-pituitary-adrenal (HPA) axis function $[9,10]$. In terms of growth in children, a nonlinear correlation between reduced growth velocity and systemic levels of corticosteroid has been identified [15]. HPA axis suppression by ICS can also be an end-point of clinical relevance in cases of adrenal insufficiency. Exogenous corticosteroids can exert HPA axis suppression, a pharmacodynamic (PD) effect, by downregulating

\section{TABLE 1 Potential local and systemic side-effects of inhaled corticosteroids}

Local side-effects

\begin{tabular}{ll}
\hline & Adrenal crisis (with insufficiency) \\
Dysphonia & Cataracts \\
Oropharyngeal candidiasis & Glaucoma \\
Pharyngitis & Decreased lower leg length in children \\
Reflex cough & Suppressed growth velocity in children \\
Sore throat & Reduced bone mineral density \\
& Osteoporosis \\
& Bone fractures \\
& Skin thinning \\
& Skin bruising
\end{tabular}

Data taken and modified from [9] adrenocorticotropic hormone production by the same feedback inhibition loops that control endogenous glucocorticoid production, thus leading to adrenal suppression and decreased cortisol levels [16]. The extent of HPA-axis suppression is dependent upon the dose, duration of treatment and timing of corticosteroid administration [17]. Serum cortisol area under the curve (24-h) and the low- and high-dose tetracosactide stimulation tests are standard assays for basal cortisol secretion and HPA-axis responsiveness, respectively [16, 18, 19].

The safety and efficacy profile of an ICS is known to be influenced by the pharmacokinetic (PK) properties and associated PD effects of the drug [20,21]. The efficacy of an ICS is determined by those PK characteristics that are critical for the anti-inflammatory activity of the ICS in the effective control of asthma, whereas the safety of an ICS is improved by those PK characteristics that reduce local and systemic exposure to active drug. The objectives of the present review are to identify and discuss specific PK and PD factors that influence the efficacy and safety profiles of ICS, demonstrate the clinical relevance of these PK and PD factors to efficacy and local and systemic side-effects, and compare the PK data pertaining to currently available ICS.

\section{PHARMACOKINETIC AND PHARMACODYNAMIC FACTORS OF INHALED CORTICOSTEROIDS}

In order to better understand the effect of PK and PD parameters on the safety of ICS, it is helpful to briefly review the fate of an ICS, as represented schematically in figure 1 [21]. On inhalation, a significant portion of the dose is deposited in the mouth and pharynx, where it has the potential to exert local side-effects. This portion of the dose, if not rinsed out of the mouth, may be swallowed and subsequently absorbed from the gastrointestinal tract [22]. Drug that is absorbed from the gastrointestinal tract and that escapes inactivation by firstpass metabolism in the liver enters the systemic circulation unchanged, potentially causing extrapulmonary side-effects [21]. The portion of the ICS dose that is delivered to the lungs exerts the desired pharmacological effect; however, much of the dose that reaches the airways can subsequently be absorbed into the general circulation through the pulmonary vasculature, where it has the potential to exert systemic side-effects [22].

Various PK and PD parameters, including bioavailability, receptor-binding and half-life, contribute to the safety and efficacy profile of an ICS [21, 23]. Therefore, the delicate balance between safety and efficacy factors must be considered in the development of corticosteroids for the treatment of asthma. For example, an ICS with a high proportion of the dose deposited in the lung (pulmonary bioavailability) is likely to show enhanced efficacy, but it also exhibits greater potential for systemic side-effects because much of the drug deposited in the lung reaches the circulation (systemic bioavailability). Higher receptor-binding affinities and longer half-lives also correlate positively with both increased efficacy and increased potential for systemic side-effects [23]. Parameters such as on-site activation, clearance and protein-binding contribute significantly to the safety profile of an ICS, whereas lipid conjugation, although important for efficacy, has less significance for safety. The PD and PK parameters and comparative data of currently available ICS and their clinical relevance to safety are described below. 


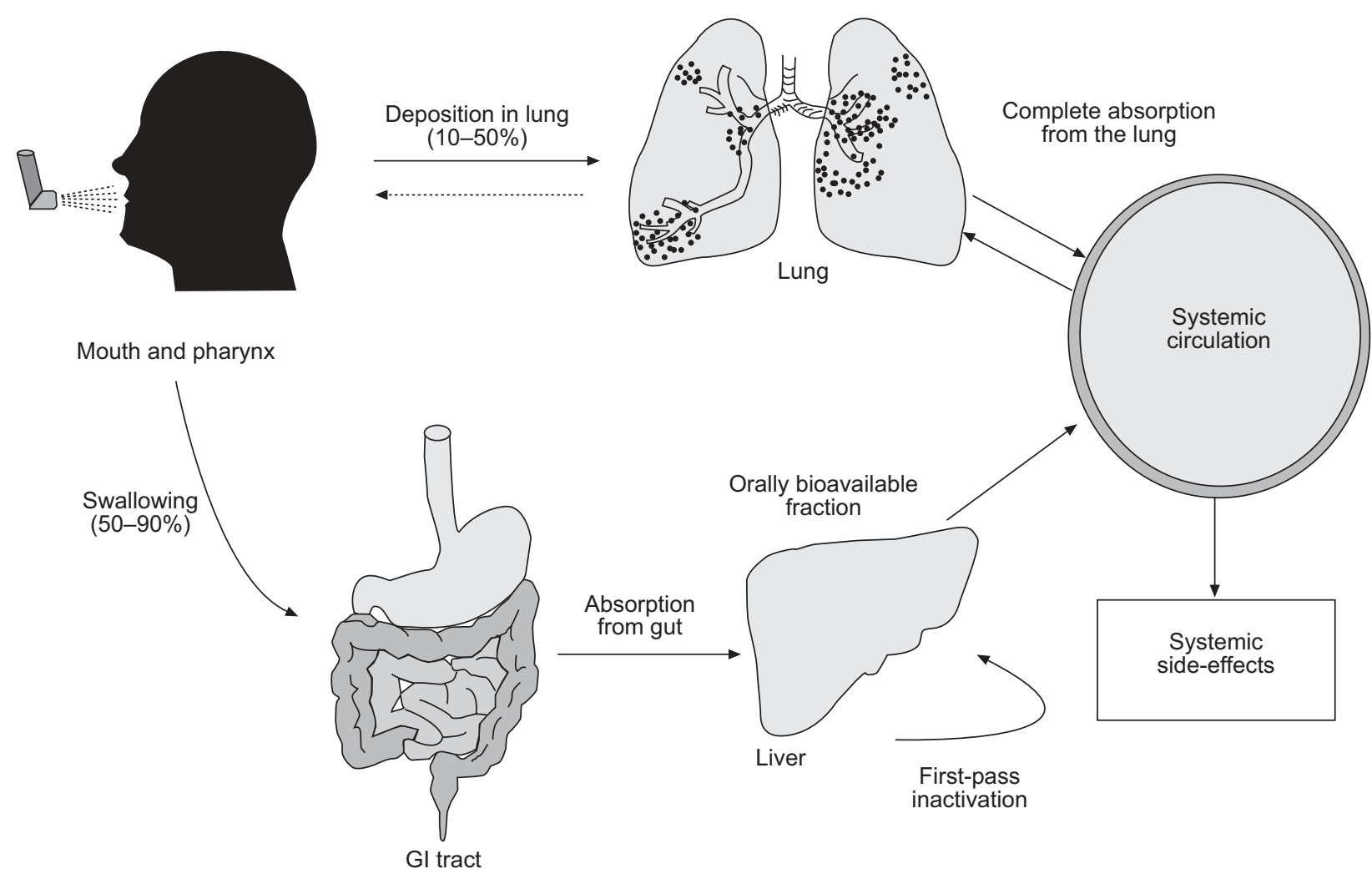

FIGURE 1. Schematic representation of the fate of an inhaled corticosteroid. Gl: gastrointestinal. From [21].

\section{Receptor-binding affinity}

The pharmacological effects of ICS are mediated through glucocorticoid receptors, widely expressed in most cell types throughout the body. Receptor-binding affinity is the only PD parameter that varies between different corticosteroids. Receptor-binding affinity has implications for the clinical safety profile of an ICS, since both the positive effects in the lung and the side-effects (local and systemic) of the drug are mediated through these same widely expressed receptors. The magnitude of the receptor-binding affinity of ICS is designated relative receptor affinity (RRA) with reference to a receptor affinity of dexamethasone of 100 [21]. The RRAs of ICS currently in use are presented in table $2[21,24,25]$. Steroids with the highest RRAs $(1,200-2,200)$ include mometasone furoate $(\mathrm{MF})$; fluticasone propionate (FP); 17-beclometasone monopropionate (BMP), the active metabolite of beclometasone dipropionate (BDP); and desisobutyryl-ciclesonide (des(IC), the active metabolite of ciclesonide. However, as noted previously, high receptor-binding affinity alone does not necessarily translate to an increased therapeutic ratio or enhanced clinical effectiveness, since corticosteroid-induced receptor activation initiates increased gene transcription, decreased gene transactivation or transrepression in all tissues, enhancing anti-inflammatory activity in the lung and potentially amplifying systemic side-effects [26].

\section{Formulation and particle size}

Particle size is an important determinant of the proportion of ICS that is deposited in the lower airways relative to the oropharyngeal cavity. The smallest airways have an internal perimeter of $\leqslant 2 \mu \mathrm{m}$ [27]. A particle of $<5 \mu \mathrm{m}$ is more likely to be deposited in bronchi and bronchioles compared to particles of $>5 \mu \mathrm{m}$, which are often deposited in the mouth and throat [28]. Larger particles $(>5 \mu \mathrm{m})$, which are more likely to be deposited in the oropharyngeal cavity, can cause local sideeffects, such as oral candidiasis and hoarseness [10, 29-31]. Particle size, therefore, affects both the efficacy and safety profiles of ICS.

Particle size distribution varies considerably between currently available ICS formulations (fig. 2) [32-36]. Among the ICSs in this comparison, FP delivered by DPI has the largest mass median aerodynamic diameter (MMAD; $>6 \mu \mathrm{m}$ ), followed by budesonide (BUD) DPI $(>2.5 \mu \mathrm{m})$. Ciclesonide and BDP delivered by hydrofluoroalkane (HFA) MDI, as solution aerosol formulations, have the smallest particles (MMAD of $<2 \mu \mathrm{m})$. The particle size of the mometasone formulation used in the HFA-MDI is not known.

Formulations of ICS in solution and suspension with HFA propellant have gradually replaced chlorofluorocarbon (CFC) formulations because of the effect of CFCs on the ozone layer. HFA solution aerosol formulations (e.g. BDP, flunisolide and ciclesonide) exist as extra-fine aerosols that penetrate more effectively into the peripheral lung, whereas HFA suspension formulations (e.g. FP and MF) retain the same particle size and lung deposition as the CFC formulations [37]. In a comparative study of BDP-HFA and BDP-CFC, 55-60\% of the BDP-HFA dose (ex-actuator) was deposited in the lungs, with $29-30 \%$ deposited in the oropharynx [38]. In contrast, deposition of the BDP-CFC dose was $4-7 \%$ in the lungs and $90-94 \%$ in the 


\begin{tabular}{|c|c|}
\hline $\begin{array}{ll}\text { TABLE } 2 & \text { Relative binding af } \\
\text { to the glucocortic }\end{array}$ & $\begin{array}{l}\text { f inhaled corticosteroids } \\
\text { eptor }\end{array}$ \\
\hline & Relative receptor affinity \\
\hline Cortisol & 10 \\
\hline Ciclesonide & 12 \\
\hline Beclometasone dipropionate & 53 \\
\hline Beclometasone & 76 \\
\hline Dexamethasone (reference) & 100 \\
\hline Flunisolide & 180 \\
\hline Triamcinolone acetonide & 233 \\
\hline Budesonide & 935 \\
\hline Desisobutyryl-ciclesonide & 1200 \\
\hline 17-beclometasone monopropionate & 1345 \\
\hline Fluticasone propionate & 1800 \\
\hline Mometasone furoate & 2200 \\
\hline
\end{tabular}

oropharynx. In another study, lung deposition of FP-CFC was $12-13 \%$, with $72-78 \%$ deposited in the oropharynx [34]. For MF-HFA, lung deposition of $7.4-24.5 \%$ and oropharyngeal deposition of $79.1 \%$ has been reported [39]. In addition, studies have indicated a lung deposition of $15-18 \%$ with BUD-MDI and 15-28\% with BUD-DPI [40, 41].

The ciclesonide HFA-MDI delivers small particles (MMAD of $1.1-2.1 \mu \mathrm{m})$ and achieves delivery of $>50 \%$ of the inhaled exactuator ciclesonide dose to the lung $34 \%$ in the peripheral airway, $36 \%$ in the middle airway and $30 \%$ in the central airway) in healthy subjects [42]. Similar results were obtained in asthmatic patients [43]. This minimises the oropharyngeal deposition of ciclesonide to $\sim 39 \%$, which compares favourably with other ICS $[42,43]$. In a study performed in 18 healthy subjects, the oropharyngeal deposition of ciclesonide $(800 \mu \mathrm{g}$ ex-valve) and des-CIC was demonstrated to be less than half that of BUD $(800 \mu \mathrm{g})$. Furthermore, the concentration of desCIC in the oropharynx was 25-fold lower than that of BUD [44]. In a similar study comparing ciclesonide ( $800 \mu \mathrm{g}$ ex-valve) and FP $(1,000 \mu \mathrm{g})$ in patients with asthma, the oropharyngeal deposition of ciclesonide and des-CIC together was approximately half that of FP, and the deposition of des-CIC was only $8 \%$ of that of FP [45]. This lower oropharyngeal deposition and minimal activation to des-CIC leads to a reduced occurrence of local side-effects (e.g. oral candidiasis) [46], while maintaining high (52\%) lung deposition [42, 43].

\section{Bioavailability}

Although ICS are intended to provide localised therapy in the lungs, the proportion of the dose that is swallowed is available for systemic absorption from the gastrointestinal tract (oral bioavailability), as is the proportion delivered to and subsequently absorbed by the lungs (pulmonary bioavailability). Therefore, the blood concentration of an ICS is a function of the sum of its pulmonary and orally absorbed fractions [21]. Since the proportion of the ICS dose that is absorbed orally may provide limited therapeutic benefit but may increase the potential for systemic side-effects, it is advantageous for the oral bioavailability of an ICS to be relatively low. The oral

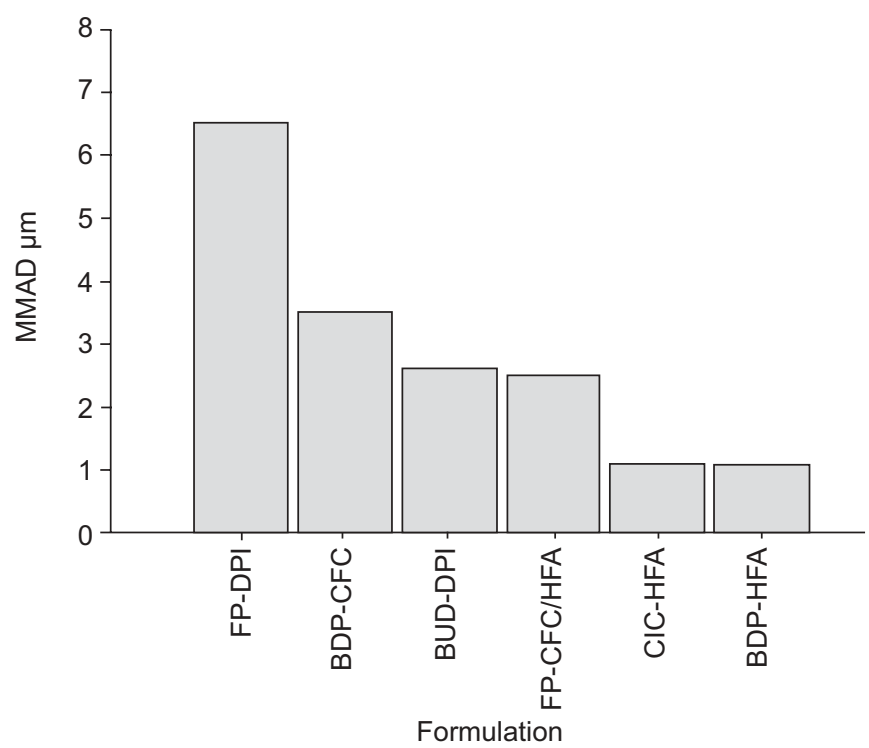

FIGURE 2. Comparative particle size of inhaled corticosteroids. Diameter is expressed as mass median aerodynamic diameter (MMAD). FP: fluticasone propionate; DPI: dry-powder inhaler; BDP: beclometasone dipropionate; CFC: chlorofluorocarbon; Bud: budesonide; HFA: hydrofluoroalkane; CIC: ciclesonide. Data from [32-35].

bioavailability of currently available ICS varies widely, from $<1 \%$ for ciclesonide, MF and FP, to $26 \%$ for BMP (table 3 ) [21, 35, 46-53].

The bioavailability of an ICS has obvious implications for the clinical safety profile of the drug. High pulmonary bioavailability, although required for efficacy, also increases systemic absorption and the potential for unwanted side-effects. High oral bioavailability of an ICS is detrimental to safety because

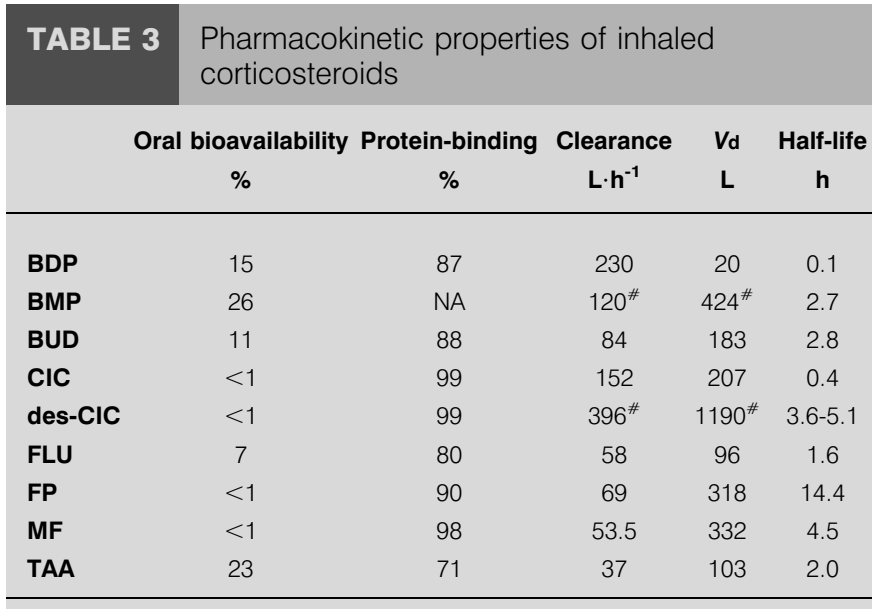

Vd: volume of distribution; BDP: beclometasone dipropionate; BMP: beclometasone monopropionate; BUD: budesonide; CIC: ciclesonide; des-CIC desisobutyryl-CIC; FLU: flunisolide; FP: fluticasone propionate; MF: mometasone furoate; TAA: triamcinolone acetonide; NA: not available. \#: apparent maximum approximation based on complete conversion of the parent compound. Data taken and collated from [21, 35, 46-53]. 
systemic absorption and the potential for unwanted sideeffects increase.

\section{On-site activation}

Many ICS, including FP and BUD, are inhaled in their pharmacologically active form, whereas other ICS, such as ciclesonide and BDP, are inhaled as inactive compounds that are converted into their active metabolites, des-CIC and 17-BMP, respectively, by esterases located in the lung epithelium [21, 23, 54, 55]. More than $90 \%$ of 17 -BMP is further metabolised to the pharmacologically less-active beclometasone [56]. The bioactivation of ciclesonide is illustrated in figure 3. Ciclesonide has a much lower RRA (12) than des-CIC $(1,200)$, and is, therefore, virtually pharmacologically inactive [24]. Studies have indicated that bioactivation of ciclesonide within the oropharynx is very low, resulting in lower amounts of active drug in the oropharyngeal region compared with BUD and FP [44, 45].

\section{Pulmonary residence time}

Lipophilicity and lipid conjugation are two distinct PK parameters that can affect the absorption rate of an ICS across pulmonary membranes and influence retention time in the lung. Slow absorption out of the lungs or pulmonary retention mechanisms enhance the anti-inflammatory effects of the drug without a corresponding increase in unwanted systemic sideeffects [26]. The pulmonary residence time of an ICS is expressed as the mean absorption time, which represents the mean time required for a molecule of the drug to be absorbed into the systemic circulation. For example, the reported mean absorption times of BUD and FP are 1.8 and $4.9 \mathrm{~h}$, respectively [57]. The contribution of lipophilicity and lipid conjugation to pulmonary residence time and their implications for the safety profile of an ICS are discussed briefly below.

\section{Lipophilicity}

The lipophilicity of an ICS, which facilitates the passage of the drug through the phospholipid bilayer of cell membranes, correlates positively with the pulmonary retention time and volume of distribution of the drug [58]. The lipophilicity of ICSs and their active metabolites varies widely: ciclesonide $\begin{array}{llll}\text { (relative lipophilicity } & 4)>\mathrm{FP} & \text { (3.2) }>\mathrm{MF} & \text { (2.0) }>\text { des-CIC }\end{array}$ (2.5) $>$ BUD (1) [59]. Lipophilic side chains added to the D-ring of an ICS slow the dissolution of the drug in the aqueous bronchial fluid and aid the passage of the drug across cellular membranes to the glucocorticoid receptors in the interior of the

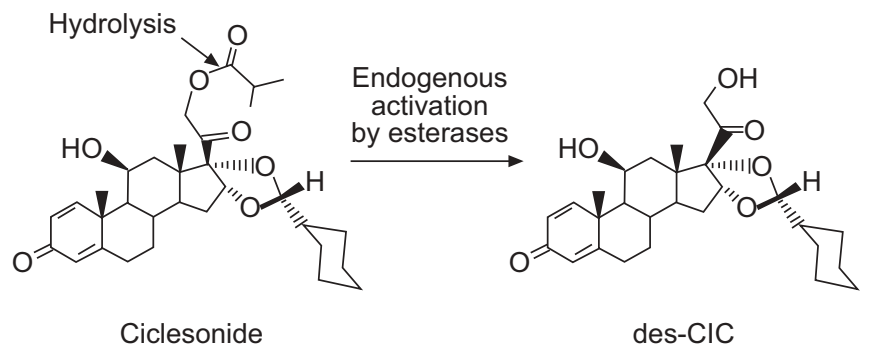

FIGURE 3. Bioactivation of ciclesonide. The arrow indicates the ester bond that is hydrolysed by cellular esterases to convert the parent compound, ciclesonide, into the active metabolite, desisobutyryl-ciclesonide (des-CIC). cell. However, lipophilicity may also alter the distribution of the ICS after systemic absorption, which may permit the drug to accumulate in other body tissues and may increase the potential for systemic side-effects.

\section{Lipid conjugation}

Lipid conjugation, or fatty acid esterification, occurs when a reversible chemical bond is formed between the ICS and fatty acids in the pulmonary cells. For lipid conjugation to occur, the ICS must have a steric-hindrance-free hydroxyl group present at the carbon 21 position [60]. Although 17-BMP, BUD, desCIC, flunisolide and triamcinolone acetonide (TAA) have a hydroxyl group at the carbon 21 position, lipid conjugation has been reported only for BUD [61], TAA [62] and des-CIC [54]. Des-CIC forms highly lipophilic fatty acid conjugates (des-CIC-oleate) that are detectable in the rat lung for up to $\sim 27 \mathrm{~h}$ after administration of ciclesonide [54]. Furthermore, des-CIC-oleate is five times more lipophilic than BUD-oleate in rat lung tissue [56]. For beclometasone, reports have indicated no formation, or only trace amounts, of lipid conjugates [56, 63]. FP does not form fatty acid conjugates [63]. MF has a furoate moiety at the carbon 17 position which enhances its lipophilicity, but the effect of this moiety on lipid conjugation of MF has not been reported [64].

Following esterification, drug-lipid complexes associate with the cell membrane and are retained. The reversibly bound drug is available for binding to glucocorticoid receptors [65]. Therefore, lipid conjugation, in effect, provides a slow-release reservoir of the ICS in the target tissue and prolongs lung residence time. This prolonged residence time may confer the ability of once-daily dosing and lessen the potential for systemic side-effects by minimising the concentration of free drug in the circulation.

\section{Protein-binding}

Protein-binding of an ICS can occur in intracellular and extracellular fluids and in the circulation. This protein-binding, generally to albumin, is rapid and reversible. The extent of protein-binding ranges 71-99\% among currently available ICS (table 3) $[21,35,46-53,66]$. The degree of protein-binding of these rapidly metabolised corticosteroids controls their unbound systemic concentrations and limits their systemic side-effects, since only the free drug is pharmacologically active. Therefore, high protein-binding of an ICS effectively reduces the potential for systemic side-effects. Both ciclesonide and des-CIC are highly protein-bound (99\%) in the systemic circulation, thereby reducing systemic exposure to des-CIC and resulting in minimal cortisol suppression [46]. Furthermore, in a recent study of the PK of $\left[{ }^{14} \mathrm{C}\right]$ ciclesonide after oral and intravenous administration, ciclesonide was not detected in any serum samples after oral administration, and the systemic bioavailability of des-CIC was $<1 \%$ [49].

\section{Inhaled corticosteroid metabolism and excretion from the body}

The metabolism and excretion of a corticosteroid are critical contributing factors to its systemic side-effects. Generally, faster metabolism leads to lower concentrations and reduced opportunity for systemic side-effects. Half-life is more difficult to interpret; an ICS with a long half-life that is present at low 
TABLE 4 Safety characteristics of an ideal inhaled corticosteroid

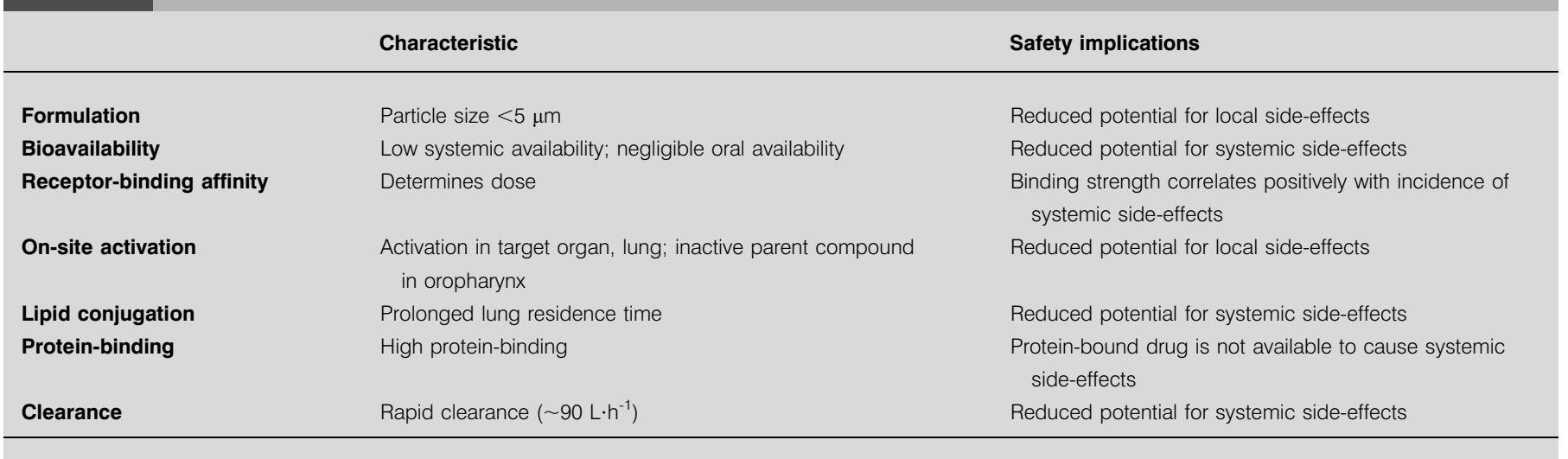

concentrations will probably have a better safety profile than an ICS with a short half-life that is present at high concentrations. The elimination half-lives of currently available ICS range 1.6-14.4 $\mathrm{h}$ (table 3) [21, 35, 46-53].

ICSs are rapidly cleared by multiple organs, primarily the liver, after systemic absorption. The maximum clearance rate by the liver is $\sim 90 \mathrm{~L} \cdot \mathrm{h}^{-1}$, which is the rate of hepatic blood flow [21]. Most currently available ICS show similar systemic clearance rates: TAA $37 \mathrm{~L} \cdot \mathrm{h}^{-1}$; flunisolide $58 \mathrm{~L} \cdot \mathrm{h}^{-1}$; FP $69 \mathrm{~L} \cdot \mathrm{h}^{-1}$; MF $53.5 \mathrm{~L} \cdot \mathrm{h}^{-1}$ : and BUD $84 \mathrm{~L}^{-1} \mathrm{~h}^{-1}$ [21]. The apparent clearance of des-CIC is $396 \mathrm{~L} \cdot \mathrm{h}^{-1}$ (assuming complete conversion of ciclesonide to its active form) [46], and appears to involve additional mechanisms of clearance by other organs in addition to the liver.

\section{MODERN AND EMERGING INHALED CORTICOSTEROIDS}

The PK and PD factors reviewed herein demonstrate that a delicate balance between safety and efficacy considerations must be achieved in the development of new corticosteroids for the treatment of asthma. An ideal ICS would incorporate PK parameters that minimise the potential for side-effects while maximising potential efficacy (table 4).

Currently available ICS are good controller medications for asthma. They possess some ideal PK and PD properties. However, very few ICS possess all of these ideal characteristics. Although FP demonstrates high receptor-binding affinity, careful monitoring of children is necessary to protect against adrenal suppression, particularly when high-dose FP therapy is necessary $[67,68]$. Moreover, FP is highly lipophilic [59], but, unlike ciclesonide and BUD, does not form fatty acid conjugates [63]. Therefore, unlike BUD and ciclesonide, which can be administered once daily, FP must be administered twice daily.

MF shows a high receptor-binding affinity and high proteinbinding (98\%) [25] and exhibits a large volume of distribution, high lipophilicity, oral bioavailability and first-pass metabolism similar to that of FP $[20,64]$. Although initial PK studies suggested that the bioavailability of MF was $<1 \%$ [64], its systemic activity may be higher, potentially due to the presence of a number of active metabolites that are usually not detected [20,69-71]. Therefore, the clinical relevance of the
PK and PD characteristics of MF are still not clear. Notwithstanding, MF has been shown to be effective in the treatment of persistent asthma [72-74], although HPAaxis suppression remains a safety concern with MF treatment $[70,71]$.

Although the RRA of BUD is lower than those of MF, FP and des-CIC [21, 24, 25], clinical studies have demonstrated that BUD has a good safety profile and is effective in the treatment of asthma [75-77]. Moreover, Bud forms fatty acid conjugates, thus allowing for once-daily dosing $[61,63]$. However, because the lipid conjugates formed by Bud are less lipophilic than those formed by des-CIC [56], the overall retention of lipid conjugates of Bud may be lower than that of des-CIC.

The novel ICS ciclesonide shows high glucocorticoid-receptorbinding, lipid conjugation, high lipophilicity, low oral bioavailability, high systemic clearance and high plasmaprotein-binding. The efficacy of once-daily dosing with ciclesonide has been indicated in a number of clinical trials in children, adolescents and adults with asthma in comparison with placebo, BUD and FP [78-81]. Furthermore, several clinical trials have demonstrated a low incidence of oropharyngeal candidiasis and no appreciable adverse effect on endogenous cortisol secretion after short- and long-term treatment of asthma with ciclesonide at doses ranging 80 $1,280 \mu \mathrm{g}$ (ex-actuator) [82-88].

In summary, the pharmacokinetic and pharmacodynamic features of the inhaled corticosteroids reviewed herein demonstrate a delicate balance between safety and efficacy considerations and that significant differences exist in the pharmacokinetic and pharmacodynamic characteristics of the currently available inhaled corticosteroids. These differences warrant careful consideration when used in clinical practice.

\section{REFERENCES}

1 Georgitis JW. The 1997 Asthma Management Guidelines and therapeutic issues relating to the treatment of asthma. Chest 1999; 115: 210-217.

2 Masoli M, Fabian D, Holt S, Beasley R. Global Burden of Asthma. http://www.ginasthma.com/ReportItem.asp?11= $2 \& 12=2$. Date accessed: October 252005. 
3 Umland SP, Schleimer RP, Johnston SL. Review of the molecular and cellular mechanisms of action of glucocorticoids for use in asthma. Pulm Pharmacol Ther 2002; 15: $35-50$.

4 Pelaia G, Vatrella A, Cuda G, Maselli R, Marsico SA. Molecular mechanisms of corticosteroid actions in chronic inflammatory airway diseases. Life Sci 2003; 72: 1549-1561.

5 Barnes PJ, Adcock IM. How do corticosteroids work in asthma? Ann Intern Med 2003; 139: 359-370.

6 Yudt MR, Cidlowski JA. The glucocorticoid receptor: coding a diversity of proteins and responses through a single gene. Mol Endocrinol 2002; 16: 1719-1726.

7 Barnes PJ. Effect of corticosteroids on airway hyperresponsiveness. Am Rev Respir Dis 1990; 141: 70-76.

8 Sont JK, Willems LN, Bel EH, van Krieken JH, Vandenbroucke JP, Sterk PJ, the AMPUL Study Group. Clinical control and histopathologic outcome of asthma when using airway hyperresponsiveness as an additional guide to long-term treatment. Am J Respir Crit Care Med 1999; 159: 1043-1051.

9 Kelly HW, Nelson HS. Potential adverse effects of the inhaled corticosteroids. J Allergy Clin Immunol 2003; 112: 469-478.

10 Hanania NA, Chapman KR, Kesten S. Adverse effects of inhaled corticosteroids. Am J Med 1995; 98: 196-208.

11 Postma DS, Sevette C, Martinat Y, Schlösser N, Aumann J, Kafé $\mathrm{H}$. Treatment of asthma by the inhaled corticosteroid ciclesonide given either in the morning or evening. Eur Respir J 2001; 17: 1083-1088.

12 Williamson IJ, Matusiewicz SP, Brown PH, Greening AP, Crompton GK. Frequency of voice problems and cough in patients using pressurized aerosol inhaled steroid preparations. Eur Respir J 1995; 8: 590-592.

13 Bender B, Milgrom H, Rand C. Nonadherence in asthmatic patients: is there a solution to the problem? Ann Allergy Asthma Immunol 1997; 79: 177-186.

14 Boulet L-P. Perception of the role and potential side effects of inhaled corticosteroids among asthmatic patients. Chest 1998; 113: 587-592.

15 Daley-Yates PT, Richards DH. Relationship between systemic corticosteroid exposure and growth velocity: development and validation of a pharmacokinetic/pharmacodynamic model. Clin Ther 2004; 26: 1905-1919.

16 Oelkers W. Adrenal insufficiency. N Engl J Med 1996; 335: 1206-1212.

17 Meibohm B, Hochhaus G, Rohatagi S, et al. Dependency of cortisol suppression on the administration time of inhaled corticosteroids. J Clin Pharmacol 1997; 37: 704-710. (Erratum in J Clin Pharmacol 1997; 37: 1000).

18 Martin RJ, Szefler SJ, Chinchilli VM, et al. Systemic effect comparisons of six inhaled corticosteroid preparations. Am J Respir Crit Care Med 2002; 165: 1377-1383.

19 Lipworth BJ, Seckl JR. Measures for detecting systemic bioactivity with inhaled and intranasal corticosteroids. Thorax 1997; 52: 476-482.

20 Crim C, Pierre LN, Daley-Yates PT. A review of the pharmacology and pharmacokinetics of inhaled fluticasone propionate and mometasone furoate. Clin Ther 2001; 23: $1339-1354$.

21 Derendorf $H$. Pharmacokinetic and pharmacodynamic properties of inhaled corticosteroids in relation to efficacy and safety. Respir Med 1997; 91: Suppl. A, 22-28.
22 Pedersen S, O'Byrne P. A comparison of the efficacy and safety of inhaled corticosteroids in asthma. Allergy 1997; 52: Suppl. 39, 1-34.

23 Derendorf H, Hochhaus G, Meibohm B, Mollmann H, Barth J. Pharmacokinetics and pharmacodynamics of inhaled corticosteroids. J Allergy Clin Immunol 1998; 101: Suppl. 1, S440-S446.

24 Dietzel K, Engelstätter R, Keller A. Ciclesonide: an on-siteactivated steroid. In: Hansel TT, Barnes PJ, eds. New Drugs for Asthma, Allergy and COPD. Basle, Karger, 2001; pp. 91-93.

25 Valotis A, Neukam K, Elert O, Hogger P. Human receptor kinetics, tissue binding affinity, and stability of mometasone furoate. J Pharm Sci 2004; 93: 1337-1350.

26 Kelly HW. Comparative potency and clinical efficacy of inhaled corticosteroids. Respir Care Clin N Am 1999; 5: 537-553.

27 Vanden Burgt JA, Busse WW, Martin RJ, Szefler SJ, Donnell D. Efficacy and safety overview of a new inhaled corticosteroid, QVAR (hydrofluoroalkane-beclomethasone extrafine inhalation aerosol), in asthma. J Allergy Clin Immunol 2000; 106: 1209-1226.

28 Pritchard JN. The influence of lung deposition on clinical response. J Aerosol Med 2001; 14: Suppl. 1, S19-S26.

29 Balter MS, Adams SG, Chapman KR. Inhaled beclomethasone dipropionate improves acoustic measures of voice in patients with asthma. Chest 2001; 120: 1829-1834.

30 Borgstrom L. The pharmacokinetics of inhaled hydrofluoroalkane formulations. J Allergy Clin Immunol 1999; 104: Suppl., S246-S249.

31 Toogood JH, Jennings B, Greenway RW, Chuang L. Candidiasis and dysphonia complicating beclomethasone treatment of asthma. J Allergy Clin Immunol 1980; 65: 145-153.

32 Fuller R. The Diskus: a new multi-dose powder device efficacy and comparison with Turbuhaler. J Aerosol Med 1995; 8: Suppl. 2, S11-S17.

33 Agertoft L, Pedersen S, Nikander K. Drug delivery from the Turbuhaler and Nebuhaler pressurized metered dose inhaler to various age groups of children with asthma. J Aerosol Med 1999; 12: 161-169.

34 Leach CL, Davidson PJ, Hasselquist BE, Boudreau RJ. Lung deposition of hydrofluoroalkane-134a beclomethasone is greater than that of chlorofluorocarbon fluticasone and chlorofluorocarbon beclomethasone: a cross-over study in healthy volunteers. Chest 2002; 122: 510-516.

35 Rohatagi S, Derendorf H, Zech K, Nave R, Banerji D. PK/ PD of inhaled corticosteroids: the risk/benefit of inhaled ciclesonide. J Allergy Clin Immunol 2003; 111: Suppl., S218.

36 Colice GL, Derendorf H, Shapiro GG. Inhaled Corticosteroids: is there an Ideal Therapy? www.medscape. com/viewprogram/2917_pnt. Date updated: February 20 2004. Date accessed: October 242005.

37 Zeidler M, Corren J. Hydrofluoroalkane formulations of inhaled corticosteroids for the treatment of asthma. Treat Respir Med 2004; 3: 35-44.

38 Leach CL, Davidson PJ, Boudreau RJ. Improved airway targeting with the CFC-free HFA-beclomethasone metered-dose inhaler compared with CFC-beclomethasone. Eur Respir J 1998; 12: 1346-1353. 
39 Pickering H, Pitcairn GR, Hirst PH, et al. Regional lung deposition of a technetium 99m-labeled formulation of mometasone furoate administered by hydrofluoroalkane 227 metered-dose inhaler. Clin Ther 2000; 22: 1483-1493.

40 Thorsson L, Edsbacker S, Conradson TB. Lung deposition of budesonide from Turbuhaler is twice that from a pressurized metered-dose inhaler P-MDI. Eur Respir J 1994; 7: 1839-1844.

41 Borgström L, Bondesson E, Morén F, Trofast E, Newman SP. Lung deposition of budesonide inhaled via Turbuhaler: a comparison with terbutaline sulphate in normal subjects. Eur Respir J 1994; 7: 69-73.

42 Leach CL, Bethke TD, Boudreau RJ, et al. Two-dimensional and three-dimensional imaging show ciclesonide has high lung deposition and peripheral distribution: a nonrandomized study in healthy volunteers. J Aerosol Med 2006; 19: 117-126.

43 Newman S, Salmon A, Nave R, Drollmann A. High lung deposition of ${ }^{99 \mathrm{~m}} \mathrm{Tc}$-labeled ciclesonide administered via HFA-MDI to patients with asthma. Respir Med 2006; 100: 375-384.

44 Nave R, Zech K, Bethke TD. Lower oropharyngeal deposition of inhaled ciclesonide via hydrofluoroalkane metered-dose inhaler compared with budesonide via chlorofluorocarbon metered-dose inhaler in healthy subjects. Eur J Clin Pharmacol 2005; 61: 203-208.

45 Richter K, Kanniess F, Biberger C, Nave R, Magnussen H. Comparison of the oropharyngeal deposition of inhaled ciclesonide and fluticasone propionate in patients with asthma. J Clin Pharmacol 2005; 45: 146-152.

46 Rohatagi S, Arya V, Zech K, et al. Population pharmacokinetics and pharmacodynamics of ciclesonide. J Clin Pharmacol 2003; 43: 365-378.

47 Derendorf H, Hochhaus G, Rohatagi S, et al. Pharmacokinetics of triamcinolone acetonide after intravenous, oral, and inhaled administration. J Clin Pharmacol 1995; 35: 302-305.

48 Ryrfeldt A, Andersson P, Edsbacker S, Tonnesson M, Davies D, Pauwels R. Pharmacokinetics and metabolism of budesonide, a selective glucocorticoid. Eur J Respir Dis Suppl 1982; 122: 86-95.

49 Nave R, Bethke TD, van Marle SP, Zech K. Pharmacokinetics of $\left[{ }^{14} \mathrm{C}\right]$ ciclesonide after oral and intravenous administration to healthy subjects. Clin Pharmacokinet 2004; 43: 479-486.

50 Daley-Yates PT, Price AC, Sisson JR, Pereira A, Dallow N. Beclomethasone dipropionate: absolute bioavailability, pharmacokinetics and metabolism following intravenous, oral, intranasal and inhaled administration in man. $\mathrm{Br} J$ Clin Pharmacol 2001; 51: 400-409.

51 Dickens GR, Wermeling DP, Matheny CJ, et al. Pharmacokinetics of flunisolide administered via metered dose inhaler with and without a spacer device and following oral administration. Ann Allergy Asthma Immunol 2000; 84: 528-532.

52 Sharpe M, Jarvis B. Inhaled mometasone furoate: a review of its use in adults and adolescents with persistent asthma. Drugs 2001; 61: 1325-1350.

53 Nave R, Zech K, Bliesath H, Bethke T. Differences between pharmacokinetics of ciclesonide-active principle and budesonide following repeated dose inhalations. Eur Respir J 2003; 22: Suppl. 45, 33s.

54 Nave R, Meyer W, Fuhst R, Zech K. Formation of fatty acid conjugates of ciclesonide active metabolite in the rat lung after 4-week inhalation of ciclesonide. Pulm Pharmacol Ther 2005; 18: 390-396.

55 Mutch E, Nave R, Zech K, Williams FM. Esterases involved in the hydrolysis of ciclesonide in human tissues. Eur Respir J 2003; 22: Suppl. 45, 267s-268s.

56 Nave R, Hummel R-P, , Wohlsen A, Herzog R, Zech K. The active metabolite of ciclesonide, des-isobutyryl ciclesonide, forms highly lipophilic fatty acid conjugates in precisioncut rat lung slices. Am J Respir Crit Care Med 2004; 169: A91.

57 Derendorf H, Hochhaus G, Mollmann H. Systemic disposition and effects of inhaled corticosteroids. In: Schleimer RP, O’Bryne PM, Szefler SJ, Brattsand R, eds. Inhaled Steroids in Asthma. Optimizing Effects in the Airways. New York, NY, Marcel Decker, Inc., 2002; pp. 3-33.

58 Lipworth BJ, Jackson CM. Safety of inhaled and intranasal corticosteroids: lessons for the new millennium. Drug Saf 2000; 23: 11-33.

59 Miller-Larsson A, Axelsson B-O, Brattsand R, Edsbacker S, Ingelf J. Relative lipophilicity of budesonide, fluticasone propionate, mometasone furoate, and ciclesonide. Preference of variable lipophilicity in airways versus systemic compartment. Am J Respir Crit Care Med 2003; 167: A773.

60 Tunek A, Sjödin K, Hallström G. Reversible formation of fatty acid esters of budesonide, an antiasthma glucocorticoid, in human lung and liver microsomes. Drug Metab Dispos 1997; 25: 1311-1317.

61 Miller-Larsson A, Jansson P, Runstrom A, Brattsand R. Prolonged airway activity and improved selectivity of budesonide possibly due to esterification. Am J Respir Crit Care Med 2000; 162: 1455-1461.

62 Hubbard WC, Blum AE, Bickel CA, Heller NM, Schleimer RP. Detection and quantitation of fatty acid acyl conjugates of triamcinolone acetonide via gas chromatography-electron-capture negative-ion mass spectrometry. Anal Biochem 2003; 322: 243-250.

63 Miller-Larsson A, Mattsson H, Hjertberg E, Dahlbäck M, Tunek A, Brattsand R. Reversible fatty acid conjugation of budesonide. Novel mechanism for prolonged retention of topically applied steroid in airway tissue. Drug Metab Dispos 1998; 26: 623-630.

64 Affrime MB, Cuss F, Padhi D, et al. Bioavailability and metabolism of mometasone furoate following administration by metered-dose and dry-powder inhalers in healthy human volunteers. J Clin Pharmacol 2000; 40: 1227-1236.

65 Edsbacker S, Brattsand R. Budesonide fatty-acid esterification: a novel mechanism prolonging binding to airway tissue. Review of available data. Ann Allergy Asthma Immunol 2002; 88: 609-616.

66 Rohatagi S, Luo Y, Shen L, et al. Protein binding and its potential for eliciting minimal systemic side effects with a novel inhaled corticosteroid, ciclesonide. Am J Ther 2005; 12: 201-209.

67 Todd GR, Acerini CL, Buck JJ, et al. Acute adrenal crisis in asthmatics treated with high-dose fluticasone propionate. Eur Respir J 2002; 19: 1207-1209. 
68 Kennedy MJ, Carpenter JM, Lozano RA, Castile RG. Impaired recovery of hypothalamic-pituitary-adrenal axis function and hypoglycemic seizures after high-dose inhaled corticosteroid therapy in a toddler. Ann Allergy Asthma Immunol 2002; 88: 523-526.

69 Derendorf H, Daley-Yates PT, Pierre LN, Efthimiou J. Systemic bioavailability of inhaled steroids: the importance of appropriate and comparable methodology. Eur Respir J 2001; 17: 157-158.

70 Fardon TC, Lee DK, Haggart K, McFarlane LC, Lipworth BJ. Adrenal suppression with dry powder formulations of fluticasone propionate and mometasone furoate. Am J Respir Crit Care Med 2004; 170: 960-966.

71 Lipworth BJ, Affrime MB. Mometasone furoate levels. Chest 2001; 120: 1034-1035.

72 Wardlaw A, Larivee P, Eller J, Cockcroft DW, Ghaly L, Harris AG. Efficacy and safety of mometasone furoate dry powder inhaler vs fluticasone propionate metered-dose inhaler in asthma subjects previously using fluticasone propionate. Ann Allergy Asthma Immunol 2004; 93: 49-55.

73 Bousquet J, D’Urzo A, Hebert J, et al. Comparison of the efficacy and safety of mometasone furoate dry powder inhaler to budesonide Turbuhaler. Eur Respir J 2000; 16: 808-816.

74 D'Urzo A, Karpel JP, Busse WW, et al. Efficacy and safety of mometasone furoate administered once-daily in the evening in patients with persistent asthma dependent on inhaled corticosteroids. Curr Med Res Opin 2005; 21: 1281-1289.

75 Selroos O, Edsbacker S, Hultquist C. Once-daily inhaled budesonide for the treatment of asthma: clinical evidence and pharmacokinetic explanation. J Asthma 2004; 41: 771-790.

76 Banov $\mathrm{CH}$. The role of budesonide in adults and children with mild-to-moderate persistent asthma. J Asthma 2004; 41: 5-17.

77 Berger WE. Budesonide inhalation suspension for the treatment of asthma in infants and children. Drugs 2005; 65: 1973-1989.

78 Pedersen S, Garcia ML, Manjra Al, Theron I, Engelstätter R. A comparative study of inhaled ciclesonide $160 \mu \mathrm{g}$ /day and fluticasone propionate $176 \mu \mathrm{g} /$ day in children with asthma. Ped Pulmonol 2006; 41: 954-961.
79 Buhl R, Vinkler I, Magyar P, et al. Comparable efficacy of ciclesonide once daily versus fluticasone propionate twice daily in asthma. Pulm Pharmacol Ther 2005; Epub ahead of print.

80 Boulet LP, Drollmann A, Magyar P, et al. Comparative efficacy of once-daily ciclesonide and budesonide in the treatment of persistent asthma. Respir Med 2006; 100: 785-794.

81 Ukena D, Biberger C, Steinijans V, et al. Ciclesonide is more effective than budesonide in the treatment of persistent asthma. Pulm Pharmacol Ther 2006 (in press).

82 Kaliner M, White M, Chervinsky P, et al., Ciclesonide, a novel inhaled corticosteroid (ICS), does not suppress hypothalamic-pituitary-adrenal (HPA)-axis function. World Allergy Organization Congress-XVIII ICACI. Abstract O-17-1. Cambridge, Hogrefe \& Huber Publishers, 2003; pp 33.

83 Hansel TT, Benezet O, Kafé H, et al. A multinational, 12week, randomized study comparing the efficacy and tolerability of ciclesonide and budesonide in patients with asthma. Clin Ther 2006; 28: 906-920.

84 Szefler S, Rohatagi S, Williams J, Lloyd M, Kundu S, Banerji D. Ciclesonide, a novel inhaled steroid, does not affect hypothalamic-pituitary-adrenal axis function in patients with moderate-to-severe persistent asthma. Chest 2005; 128: 1104-1114.

85 Derom E, Van De Velde V, Marissens S, Engelstätter R, Vincken W, Pauwels R. Effects of inhaled ciclesonide and fluticasone propionate on cortisol secretion and airway responsiveness to adenosine 5'monophosphate in asthmatic patients. Pulm Pharmacol Ther 2005; 18: 328-336.

86 Weinbrenner A, Hüneke D, Zschiesche M, et al. Circadian rhythm of serum cortisol after repeated inhalation of the new topical steroid ciclesonide. J Clin Endocrinol Metab 2002; 87: 2160-2163.

87 O'Connor BJ, Sips P, Biberger C, Steinijans VW, Wurst W. Management of moderate to severe bronchial asthma by ciclesonide: a 12-week study. Allergy 2002; 57: Suppl. 73, 341.

88 O'Connor BJ, Kilfeather S, Cheung D, et al. Treatment of moderate to severe asthma with ciclesonide: a long-term investigation over 52 weeks. Eur Respir J 2002; 20: Suppl. 38, 406s. 